\title{
A new reduced-complexity detection scheme for zero-padded OFDM transmissions
}

\author{
Homa Eghbali and Sami Muhaidat
}

\begin{abstract}
Recently, zero-padding orthogonal frequency division multiplexing (ZP-OFDM) has been proposed as an alternative solution to the traditional cyclic prefix (CP)-OFDM, to ensure symbol recovery regardless of channels nulls. Various ZP-OFDM receivers have been proposed in the literature, trading off performance with complexity. In this paper, we propose a novel low-complexity $(\mathrm{LC})$ receiver for ZP-OFDM transmissions and derive an upper bound on the bit error rate (BER) performance of the LC-ZP-OFDM receiver. We further demonstrate that the LC-ZP-OFDM receiver brings a significant complexity reduction in the receiver design, while outperforming conventional minimum mean-square error (MMSE)-ZP-OFDM, supported by simulation results. A modified (M)-ZP-OFDM receiver, which requires the channel state information (CSI) knowledge at the transmitter side, is presented. We show that the M-ZP-OFDM receiver outperforms the conventional MMSE-ZP-OFDM when either perfect or partial CSI (i.e., limited (SI) is available at the transmitter side.

Index Terms-Zero-padding, orthogonal frequency division multiplexing (OFDM), equalization.
\end{abstract}

\section{Introduction}

The growing demand for high data rate services for wireless multimedia and internet services has led to intensive research efforts on high-speed data transmission. A key challenge for high-speed broadband applications is the dispersive nature of frequency-selective fading channels, which causes the so-called intersymbol interference (ISI), leading to an inevitable performance degradation. An efficient approach to mitigate ISI is the use of orthogonal frequency division multiplexing (OFDM) that converts the ISI channel with additive white Gaussian noise (AWGN) into parallel ISI-free subchannels by implementing inverse fast Fourier transform (IFFT) at the transmitter and fast Fourier transform (FFT) at the receiver side [1]. It has been shown that OFDM is an attractive equalization scheme for digital audio/video broadcasting (DAB/DVB) $[2,3]$ and has successfully been applied to high-speed modems over digital subscriber lines (DSL) [4]. Recently, it has also been proposed for broadband television systems and mobile wireless local area networks such as IEEE802.11a and HIPERLAN/2 (HL2) standards [5].

\footnotetext{
* Correspondence: muhaidat@ieee.org

School of Engineering Science, Simon Fraser University, Burnaby, BC, V5A 156, Canada
}

\section{黑 Springer}

(c) 2011 Eghbali and Muhaidat; licensee Springer. This is an Open Access article distributed under the terms of the Creative Commons Attribution License (http://creativecommons.org/licenses/by/2.0), which permits unrestricted use, distribution, and reproduction in any medium, provided the original work is properly cited.
The IFFT precoding at the transmitter side and insertion of CP enable OFDM with very simple equalization of frequency-selective finite impulse response (FIR) channels. To avoid in-terblock interference (IBI) between successive FFT processed blocks, the CP is discarded and the truncated blocks are FFT processed so that the frequency-selective channels are converted into parallel flat-faded independent subchannels. In this way, the linear channel convolution is converted into circular convolution, and the receiver complexity both in equalization and the symbol decoding stages is reduced [6]. However, since each symbol is transmitted over a single flat subchannel, the multipath diversity is lost along with the fact there is no guarantee for symbol detectability when channel nulls occur in the subchannels. As an additional counter-measure, coded OFDM (C-OFDM), which is fading resilient, has been adopted in many standards [7]. Trellis-coded modulation (TCM) [8] and convolutional codes $[9,10]$ are typical choices for error-control codes. Interleaving together with TCM enjoys low-complexity Viterbi decoding while enabling a better trade off between bandwidth and efficiency. However, designing systems that achieve diversity gain equal to code length is difficult considering the standard design paradigms for TCM. Linear constellation precoded OFDM (LCP-OFDM) was further proposed in 
[11] to improve performance over fading channels, where a real orthogonal precoder is applied to maximize the minimum product distance $[12,13]$ and channel cutoff rate [14], while maintaining the transmission rate and guaranteeing the symbol recovery. In $[15,16]$, the LCP is combined with space-time block coding (STBC) and channel coding in order to effectively improve the space-time diversity order of the multiple antenna system. The aforementioned LCP systems are based on Hadamard matrices and can employ the simple MMSE linear decoder at the receiver side, which has low complexity compared to the maximum likelihood (ML) decoders currently used in such systems. Subcarrier grouping was proposed in [11] to enable the maximum possible diversity and coding gain. However, the LCP that is used within each subset of subcarriers is in general complex.

To ensure symbol recovery regardless of channel nulls, Zero-Padding (ZP) of multicarrier transmission has been proposed to replace the generally non-zero CP. Unlike CP-OFDM, ZP-OFDM guarantees symbol recovery and FIR equalization of FIR channels. Specifically, the zero symbols are appended after the IFFT processed information symbols. In such way, the single FFT required by CP-OFDM is replaced by FIR filtering in ZP-OFDM that adds to the receiver complexity. To trade-off BER performance for extra savings in complexity, two lowcomplexity equalizers for ZP-OFDM are derived in [17]. Both schemes are developed based on the circularity of channel matrix while reducing complexity by avoiding inversion of a channel dependent matrix. However, the BER performance of the two sub-optimal receivers proposed in [17] is not as good as MMSE-ZP-OFDM.

Adapting the transmitter designs to the propagation channel facilitates performance improvement. Depending on the CSI available to the transmitter, various parameters such as power levels, constellation size, and modulation are adjustable by the channel-adaptive transmissions [18]-[20]. The challenge in a wireless setting is on whether and what type of CSI can be practically made available to the transmitter, where fading channels are randomly varying. Apparently, this is less of an issue for discrete multi-tone (DMT) systems in wireline links, which has been standardized for digital subscriber line modems. In both single-input single-output (SISO) and multiple-input multiple-output (MIMO) versions of DMT, perfect CSI is assumed to be available at the transmitter [21]. Although it is reasonable for wireline links, we can only justify the assumption of perfect CSIbased adaptive transmissions developed for SISO [22] and MIMO OFDM wireless systems [23] when the fading is sufficiently slow. Since no-CSI leads to robust but rather pessimistic designs and perfect CSI is mostly impractical for wireless links, recent efforts geared toward exploitation of partial CSI-based transmissions. In this work, we assume limited CSI knowledge. In particular, we assume that only small number of channel taps are available at the transmitter.

In this paper, we propose a novel reduced-complexity receiver design for ZP-OFDM transmissions (LC-ZPOFDM) that not only outperforms MMSE-ZP-OFDM (confirmed through simulation results) but also uses low-complexity computational methods that bring a significant power saving to the proposed receiver. We show that linear processing collects both multipath and spatial diversity gains, leading to significant improvement in performance, while maintaining low complexity. Note that the proposed LC-ZP-OFDM receiver consists of two stages, i.e., a low-complexity sub-optimal MMSE receiver at first stage followed by linear operations at second stage to investigate the underlying available diversity. A M-ZP-OFDM receiver for transmitter designs based on perfect, as well as limited CSI knowledge, is further included, which significantly outperforms MMSE-ZP-OFDM. BER upper bounds are presented for the two-stage LC receiver. The performance improvement of the LC-ZP-OFDM and M-ZPOFDM receivers as compared with the conventional MMSE-ZP-OFDM receiver is further corroborated through simulation results.

The rest of this correspondence is organized as follows. We start in Section II by describing our model and assumptions. In Section III, the reduced-complexity MMSE-based receiver is proposed. Computational complexity analysis and numerical results are presented in Sections IV and V, respectively. The paper is concluded in Section VI.

\section{System model}

We consider a single-transmit single-receive antenna system over a frequency-selective fading wireless channel. The channel impulse response (CIR) of the $j$ th information block is modeled as an FIR filter with coefficients $\mathbf{h}^{j}$ $=\left[h^{j}[0], \ldots, h^{j}[L]\right]^{\mathrm{T}}$, where $L$ denotes the corresponding channel memory length. The random vectors $\mathbf{h}^{j}$ are assumed to be independent zero-mean complex Gaussian with two choices of power-delay profile: Uniform powerdelay profile with variance $1 /(L+1)$, and exponentially decaying power-delay profile $\theta\left(\tau_{k}\right)=C e^{-\tau_{k} / \tau_{r m s}}$ with delays $\tau_{k}$ that are uniformly and independently distributed [24].

The $j$ th $M \times 1$ information block $\mathbf{x}_{M}^{j}$ is IFFT precoded by the IFFT matrix to yield the time-domain block vector $\tilde{\mathbf{x}}_{z p}^{j}$ and padded by $D$ trailing zeros as follows

$$
\tilde{\mathbf{x}}_{z p}^{j}=\mathbf{Q}_{z p} \mathbf{x}_{M}^{j}
$$


where

$$
\mathbf{Q}_{z p}=\left[\mathbf{Q}_{M} \mathbf{0}_{D \times M}\right]^{H}
$$

Therefore, the received block symbol is given by

$$
\tilde{\mathbf{r}}_{z p}^{j}=\mathbf{H Q}_{z p} \mathbf{x}_{M}^{j}+\mathbf{H}_{I B I} \mathbf{Q}_{z p} \mathbf{x}_{M}^{j-1}+\tilde{\mathbf{n}}_{N}^{j}
$$

where $N=M+D ; \mathbf{H}$ is the $N \times N$ lower triangular Toeplitz filtering matrix with its first column being $\left[h^{j}\right.$ $\left.[0], \ldots, h^{j}[L] 0, \ldots, 0\right]^{\mathrm{T}} ; \mathbf{H}_{I B I}$ is the $N \times N$ upper triangular Toeplitz filtering matrix that captures the inter-block interference (IBI), with its first row being $\left[0, \ldots, 0 h^{j}[0], \ldots\right.$, $\left.h^{j}[L]\right]$; and $\tilde{\mathbf{n}}_{N}^{j}$ denotes the AWGN noise. Since $L+1 \leq$ $D$, we have $\mathbf{H}_{I B I} \mathbf{Q}_{z p}=0$, i.e., the all-zero $\mathbf{0}_{D \times M}$ matrix plays the key role in ZP-OFDM by eliminating the IBI. Having the $\mathbf{H}$ matrix partitioned between its first $M$ and last $D$ columns as $\mathbf{H}=\left[\mathbf{H}_{0}, \mathbf{H}_{z p}\right]$, the received block symbol will be given by

$$
\tilde{\mathbf{r}}_{z p}^{j}=\mathbf{H} \mathbf{Q}_{z p} \mathbf{x}_{M}^{j}+\tilde{\mathbf{n}}_{N}^{j}=\mathbf{H}_{0} \mathbf{Q}_{M}^{H} \mathbf{x}_{M}^{j}+\tilde{\mathbf{n}}_{N}^{j} .
$$

The $N \times M$ submatrix $\mathbf{H}_{0}$, which corresponds to the first $M$ columns of $\mathbf{H}$, is Toeplitz and is guaranteed to be invertible, which assures symbol recovery regardless of channel zero locations. In this case, due to its channel-irrespective symbol detectability property, ZPOFDM is able to exploit fully the underlying multipath diversity [1]. Assuming the symbols $\mathbf{x}_{M}^{j}$ are i.i.d with variance $\sigma_{x}^{2}$ and the additive white Gaussian noise $\tilde{\mathbf{n}}_{N}^{j}$ to be i.i.d with variance $\sigma_{x}^{2}$, the minimum mean-square error (MMSE) estimator of $\mathbf{x}_{M}^{j}$ is given by [17]

$$
\hat{\mathbf{x}}_{M}^{j}=\sigma_{x}^{2} \mathbf{Q}_{M} \mathbf{H}_{0}^{H}\left(\sigma_{n}^{2} \mathbf{I}_{N}+\sigma_{x}^{2} \mathbf{H}_{0} \mathbf{H}_{0}^{H}\right)^{-1} \tilde{\mathbf{r}}_{z p}^{j} .
$$

It should be emphasized that channel inversion of a $N$ $\times N$ matrix cannot be precomputed due to its dependence on channel that brings about an extra implementation cost for the MMSE-ZP-OFDM receiver. Specifically, equalization in (5) is computationally costly, as the pseudo inverse of the Toeplitz matrix $\mathbf{H}_{0}$ is required, whose precomputation is impossible due to the channel coefficients' variations from block to block. Thus, from an implementation point of view, a lowcomplexity ZP-OFDM is desirable. This observation motivates the subsequent sub-optimal low-complexity ZP-OFDM equalizers, such as ZP-OFDM-FAST-ZF/ MMSE and the ZP-OFDM-OLA [17] originating from the overlap-and-add (OLA) method. Thus, complexity is always an issue when dealing with ZP-OFDM systems, as compared to the computationally efficient FFT-based CP-OFDM systems. Its worth mentioning that ZPOFDM-OLA is analogous to CP-OFDM; thus, it incurs identical complexity and is not able to fully exploit the underlying multipath diversity. To overcome these limitations, our proposed scheme targets practical ZPOFDM receiver that exploits full multipath diversity while maintaining low complexity.

\section{Reduced-complexity mmse-based receiver}

The proposed receiver includes a suboptimal channel independent MMSE equalizer in the feed forward stage, followed by linear processing operations in the feedback stage. Specifically, the feed forward stage exploits the circulant structure of the channel matrices,

$$
\mathbf{H}^{\prime}=\mathbf{Q} \mathbf{\Lambda} \mathbf{Q}^{H}
$$

where $\mathbf{H}^{\prime}$ is $N \times N$ circulant matrices with entries $\left[\mathbf{H}^{\prime}\right]$ $k, l=\mathbf{h}((k-l) \bmod N) ; \boldsymbol{\Lambda}$ is a diagonal matrix whose $(n$, $n$ ) element is equal to the $n$th DFT coefficient of $\mathbf{h}$. Due to the trailing zeros, the last $D$ columns of $\mathbf{H}$ in (4) do not affect the received block. Thus, the Toeplitz matrix $\mathbf{H}$ can be replaced by the $N \times N$ circulant matrix $\mathbf{H}^{\prime}$ and (4) can be written as

$$
\tilde{\mathbf{r}}_{z p}^{j}=\mathbf{H} \mathbf{Q}_{z p} \mathbf{x}_{M}^{j}+\tilde{\mathbf{n}}_{N}^{j}=\mathbf{H}^{\prime} \mathbf{Q}_{z p} \mathbf{x}_{M}^{j}+\tilde{\mathbf{n}}_{N}^{j} .
$$

The $\mathbf{H}^{\prime}$ matrix takes advantage of FFT to yield a set of flat fading channels that can be equalized easily. The ZP-OFDM receiver output will be

$$
\begin{aligned}
& \mathbf{r}_{N}^{j}=\mathbf{Q}_{N} \mathbf{H}^{\prime} \mathbf{Q}_{z p} \mathbf{x}_{M}^{j}+\mathbf{n}_{N}^{j} \\
& =\mathbf{Q}_{N} \mathbf{H}^{\prime} \mathbf{Q}_{N}^{H} \mathbf{Q}_{N} \mathbf{Q}_{z p} \mathbf{x}_{M}^{j}+\mathbf{n}_{N}^{j} \\
& =\boldsymbol{\Lambda} \mathbf{Q}_{N} \mathbf{Q}_{z p} \mathbf{x}_{M}^{j}+\mathbf{n}_{N}^{j}=\mathbf{\Lambda} \mathbf{V} \mathbf{x}_{M}^{j}+\mathbf{n}_{N}^{j}
\end{aligned}
$$

where

$$
\mathbf{V}=\mathbf{Q}_{N} \mathbf{Q}_{z p} .
$$

After applying the $N$-point FFT $\mathbf{Q}_{N}$ to $\tilde{\mathbf{r}}_{z p}^{j}$, the suboptimal MMSE-ZP-OFDM receiver in the feed forward stage is formed in two steps. First, we obtain an MMSE estimator of $\hat{\mathbf{x}}_{M}^{j}$ using

$$
\begin{aligned}
\hat{\mathbf{x}}_{M}^{j} & =E\left[\mathbf{x}_{M}^{j}\left(\mathbf{r}_{N}^{j}\right)^{H}\right] \cdot E^{-1}\left[\mathbf{r}_{N}^{j}\left(\mathbf{r}_{N}^{j}\right)^{H}\right] \\
& =\sigma_{x}^{2} \mathbf{V}^{H} \boldsymbol{\Lambda}^{H}\left[\sigma_{n}^{2} \mathbf{I}+\sigma_{x}^{2} \boldsymbol{\Lambda} \mathbf{V} \mathbf{V}^{H} \boldsymbol{\Lambda}^{H}\right]^{-1} \mathbf{r}_{N}^{j} .
\end{aligned}
$$

The $(m, n)$ th element of $\mathbf{V} \mathbf{V}^{H}$ is equivalent to

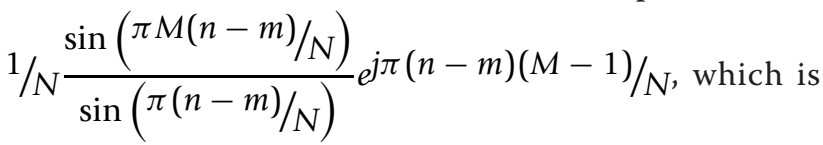
equal to $M / N$ for the diagonal elements and is negligible compared to the diagonal elements when $M / N$ is close to 1 . This is a common feature in the context of all current standardized OFDM systems, i.e., DAB and DVB. Therefore, (10) can be further simplified assuming that 


$$
\begin{gathered}
\hat{\mathbf{x}}_{M}^{j}=\sigma_{x}^{2} \frac{N}{M} \mathbf{V}^{H} \boldsymbol{\Lambda}^{H}\left[\frac{N}{M} \sigma_{n}^{2} \mathbf{I}+\sigma_{x}^{2} \boldsymbol{\Lambda} \boldsymbol{\Lambda}^{H}\right]^{-1} \mathbf{r}_{N}^{j}, \text { leading to } \\
\hat{\mathbf{x}}_{M}^{j}=\sigma_{x}^{2} \frac{N}{M} \mathbf{V}^{H} \boldsymbol{\Lambda}^{H}\left[\frac{N}{M} \sigma_{n}^{2} \mathbf{I}+\sigma_{x}^{2} \boldsymbol{\Lambda} \boldsymbol{\Lambda}^{H}\right]^{-1} \mathbf{r}_{N}^{j} .
\end{gathered}
$$

The resulting data streams detected from (11) are fed into a minimum Euclidean distance decoder, yielding $\tilde{\mathbf{x}}_{M}^{j}$, i.e., a decoded version of the transmitted symbols. The decoded signals are then fed into the feed-back stage, which is responsible for exploiting the underlying multipath diversity. Specifically, consider the generation of the matrix $\boldsymbol{\Pi}_{l}, l=0,1,2, \ldots, L$, as follows:

$$
\left[\Pi_{l}\right]_{p, q}=\left\{\begin{array}{cr}
0 & (p-q) \bmod N=l \\
{\left[\mathbf{H}^{\prime}\right]_{p, q}} & (p-q) \bmod N \neq l
\end{array}\right.
$$

where $1 \leq p, q \leq N$, then, (7) can be re-written as

$$
\tilde{\mathbf{r}}_{l}^{\prime}=\tilde{\mathbf{r}}_{z p}^{j}-\Pi_{l} \mathbf{Q}_{z p} \hat{\mathbf{x}}_{M}^{j} .
$$

Under the high SNR assumption, we can safely assume that $\mathbf{x}_{M}^{j} \approx \hat{\mathbf{x}}_{M^{*}}^{j}$. Therefore, we can write (13) as follows:

$$
\tilde{\mathbf{r}}_{l}^{\prime}=\mathbf{H}_{l} \mathbf{Q}_{z p} \mathbf{x}_{M}^{j}+\tilde{\mathbf{n}}_{N}^{j}
$$

where

$$
\left[\mathbf{H}_{l}\right]_{p, q}=\left\{\begin{array}{cc}
{\left[\mathbf{H}^{\prime}\right]_{p, q}} & (p-q) \bmod N=l \\
0 & (p-q) \bmod N \neq l .
\end{array}\right.
$$

Combining each diversity branch in (14), we have

$$
\tilde{\mathbf{z}}^{j}=\frac{\mathbf{Q}_{z p}^{H} \sum_{l=0}^{L} \mathbf{H}_{l}^{H} \tilde{\mathbf{r}}_{l}^{\prime}}{\sum_{l=0}^{L}\left\|\mathbf{H}_{l}\right\|^{2}} .
$$

The signal in (16) is then fed into a maximum likelihood decoder to recover the transmitted symbols. It is observed from (16) that the maximum achievable diversity order is given by $L+1$. This illustrates that our proposed receiver is able to fully exploit the underlying spatial and multipath diversity gains relying on simple linear processing operations only.

\section{M-ZP-OFDM RECEIVER}

In this section, we propose a M-ZP-OFDM receiver that has access to CSI at the transmitter side, such that, at the transmitter side, the zero-padded information symbols are multiplied by the set of matrices $\mathbf{C}_{2, l}$ and sent through the channel, processed by the circulant matrix $\mathbf{H}^{\prime}$ and finally multiplied by the the set of matrices $\mathbf{C}_{1, l}$ at the receiver side to exploit the underlying multipath diversity. For $l=1, \ldots, L$, the matrices $\mathbf{C}_{1, l}$ and $\mathbf{C}_{2, l}$ are generated as following [25].

$$
\begin{aligned}
\mathbf{C}_{1, l} & =\left[\begin{array}{cccc}
\frac{a_{1, l}}{\sqrt{\sum_{t=1}^{N}\left|a_{t, l}\right|^{2}}} & 0 & \cdots & 0 \\
0 & \frac{a_{2, l}}{\sqrt{\sum_{l=1}^{N}\left|a_{t, l}\right|^{2}}} & \cdots & \vdots \\
\vdots & \cdots & \ddots & 0 \\
0 & 0 & \cdots & \frac{a_{N, l}}{\sqrt{\sum_{l=1}^{N}\left|a_{t, l}\right|^{2}}}
\end{array}\right] \\
\mathbf{C}_{2, l} & =\left[\begin{array}{cccc}
\frac{\sqrt{\sum_{t=1}^{N}\left|a_{t, l}\right|^{2}}}{\sum_{k=1}^{L} a_{1, k}} & 0 & \cdots & 0 \\
0 & \frac{\sqrt{\sum_{t=1}^{N}\left|a_{t, l}\right|^{2}}}{\sum_{k=1}^{L} a_{2, k}} & \cdots & \vdots \\
\vdots & \ldots & \ddots & 0 \\
0 & 0 & \cdots & \frac{\sqrt{\sum_{t=1}^{N}\left|a_{t, l}\right|^{2}}}{\sum_{k=1}^{L} a_{N, k}}
\end{array}\right],
\end{aligned}
$$

where

$$
\boldsymbol{\Lambda}_{l}=\left\{\begin{array}{cccc}
a_{1, l} & 0 & \cdots & 0 \\
0 & a_{2, l} & \cdots & \vdots \\
\vdots & \cdots & \ddots & 0 \\
0 & 0 & \cdots & a_{N, l}
\end{array}\right\} .
$$

Note that $\mathbf{C}_{1, l}$ and $\mathbf{C}_{2, l}$ are built such that $\boldsymbol{\Lambda}_{l}=\mathbf{C}_{1, l}$ $\boldsymbol{\Lambda} \mathbf{C}_{2, l}$, where $\boldsymbol{\Lambda}_{l}=\mathbf{Q}^{H} \mathbf{H}_{l} \mathbf{Q}$. Using this property, we can drive their corresponding circulant matrices $\boldsymbol{\Psi}_{1, l}$ and $\boldsymbol{\Psi}_{2}$, $l$ using $\boldsymbol{\Psi}_{1, l}=\mathbf{Q} \mathbf{C}_{1, l} \mathbf{Q}^{H}$ and $\boldsymbol{\Psi}_{2, l}=\mathbf{Q} \mathbf{C}_{2, l} \mathbf{Q}^{H}$. Thus exploiting the circulant structure of these matrices we have

$$
\mathbf{H}_{l}=\boldsymbol{\Psi}_{1, l} \mathbf{H}^{\prime} \boldsymbol{\Psi}_{2, l} .
$$

Thus, multiplying the zero-padded symbols at the transmitter side with $\boldsymbol{\Psi}_{2, l}$ and the output at the receiver side by $\boldsymbol{\Psi}_{1, l}$, for each diversity branch we can rewrite (7) as following

$$
\begin{aligned}
& \mathbf{r}_{c, l}=\mathbf{H}^{\prime} \boldsymbol{\Psi}_{2, l} \mathbf{Q}_{z p} \mathbf{x}_{M}^{j}+\mathbf{n}_{N}^{j} \\
& \mathbf{r}_{c, l}^{\prime}=\boldsymbol{\Psi}_{1, l} \mathbf{r}_{c, l}=\boldsymbol{\Psi}_{1, l} \mathbf{H}^{\prime} \boldsymbol{\Psi}_{2, l} \mathbf{Q}_{z p} \mathbf{x}_{M}^{j}+\boldsymbol{\Psi}_{1, l} \mathbf{n}_{N}^{j} \\
& =\mathbf{H}_{l} \mathbf{Q}_{z p} \mathbf{x}_{M}^{j}+\boldsymbol{\Psi}_{1, l} \mathbf{n}_{N}^{j}
\end{aligned}
$$

Note that for $l=1, \ldots, L+1$, both $\mathbf{C}_{1, l}$ and $\boldsymbol{\Psi}_{1, l}$ have unit power and thus do not lead to noise enhancement. More importantly, it can be seen that (20) is similar to (14) in LC-ZP-OFDM proposed in Section III, in the sense that we are exploiting the underlying multipath diversity from each diversity branch.

\section{Analytical bounds on the ber performance for the low-complexity receiver}

The two-stage receiver consists of the suboptimal MMSE-ZP-OFDM at the first stage and the linear operations at the second stage. The BER can then be written as 


$$
\begin{aligned}
p_{e}= & \left(p_{e-1^{\text {st }} \text { Stage }} \cap p_{e-2^{\text {nd }} \text { Stage }}\right) \\
& +\left(\left(1-p_{e-1^{s t} \text { Stage }}\right) \cap p_{e-2^{\text {nd }} \text { Stage }}\right),
\end{aligned}
$$

where $p_{e-1^{s t} \text { Stage }}$ stands for the probability of decoding the symbols erroneously at the first stage and $p_{e-2^{n d} \text { Stage }}$ stands for the probability of decoding the symbols erroneously at the second stage. $p_{e}$ can be further simplified as following

$$
\begin{aligned}
p_{e}= & \left(p_{e-2^{n d} \text { Stage }} \mid p_{e-1^{\text {st }} \text { Stage }}\right) p_{e-1^{\text {st }} \text { Stage }} \\
& +\left(p_{e-2^{\text {nd }} \text { Stage }} \mid 1-p_{e-1^{s t} \text { Stage }}\right)\left(1-p_{e-1^{\text {st }} \text { Stage }}\right) \\
= & {\left[\left(p_{e-2^{n d} \text { Stage }} \mid p_{e-1^{\text {st }} \text { Stage }}\right)-\left(p_{e-2^{\text {nd }} \text { Stage }} \mid 1-p_{e-1^{\text {st }} \text { Stage }}\right)\right] } \\
& \times p_{e-1^{\text {st }} \text { Stage }}+\left(p_{e-2^{\text {nd }} \text { Stage }} \mid 1-p_{e-1^{\text {st }} \text { Stage }}\right)
\end{aligned}
$$

In order to find each of $p_{e-1^{s t} \text { Stage, }}$ $p_{e-2^{n d} \text { Stage }} \mid\left(1-p_{e-1^{s t} \text { Stage }}\right)$, and $\left(p_{e-2^{n d} \text { Stage }} \mid p_{e-1^{\text {st }} \text { Stage }}\right)$, we resort to the conventional Chernoff bound on the BER performance. As mentioned earlier, through the MMSE criterion, the mean-squared error (MSE) is minimized, which can be expressed as following [25]

$$
\begin{aligned}
\varepsilon_{\text {MMSE }} & =E\left[\operatorname{tr}\left(\left(\hat{\mathbf{x}}_{M}^{j}-\mathbf{x}_{M}^{j}\right)\left(\hat{\mathbf{x}}_{M}^{j}-\mathbf{x}_{M}^{j}\right)^{H}\right)\right] \\
& =\left[\operatorname{tr}\left(\frac{1}{\sigma_{d}^{2}} \mathbf{I}_{M}+\frac{1}{\sigma_{n}^{2}} \mathbf{V}^{H} \mathbf{\Lambda}^{H} \mathbf{\Lambda} \mathbf{V}\right)\right]^{-1} \\
& \approx \underbrace{\left[\operatorname{tr}\left(\frac{1}{\sigma_{d}^{2}} \mathbf{I}_{M}+\frac{M}{\sigma_{n}^{2} N} \mathbf{Q}_{z p}^{H} \mathbf{H}^{{ }^{H}} \mathrm{H}^{\prime} \mathbf{Q}_{z p}\right)\right]^{-1}}_{\text {as is considered in the 1 } 1^{\text {st }} \text { stage sub-optimal receiver }} \\
= & \frac{N \sigma_{n}^{2}}{M}\left[\frac{N \sigma_{n}^{2}}{M \sigma_{d}^{2}}+\sum_{l=1}^{L}|\mathbf{h}(l)|^{2}\right]
\end{aligned}
$$

The upper bound for the conditional $p_{e-1^{s t} \text { Stage }}$ can be then written as

$$
\begin{aligned}
& p_{e-1^{s t} \text { Stage }} \leq \exp \left(\frac{-d_{c}^{2} \sigma_{d}^{2}\left(1-\frac{\varepsilon_{\mathrm{MMSE}}}{\sigma_{d}^{2}}\right)}{4 \varepsilon_{\mathrm{MMSE}}}\right) \\
& =\exp \left(\frac{d_{c}^{2}}{4}\left(1-\frac{\sigma_{d}^{2}}{\varepsilon_{\mathrm{MMSE}}}\right)\right) \\
& =\exp \left(-\frac{M d_{c}^{2} \sigma_{d}^{2}}{4 N \sigma_{n}^{2}} q\right),
\end{aligned}
$$

where $q=\sum_{l=1}^{L}|\mathbf{h}(l)|^{2}$ whose pdf is denoted by $p(q)$ that has a chi-square distribution with $2 L$ degrees of freedom, and $d_{c}^{2}$ stands for the minimum squared distances for different modulation types that are summarized in Table 1. Note that for M-PSK modulation, $d_{c}^{2}=\left|1-e^{-j 2 \pi / M}\right|^{2}$. The average BER is thus upper bounded by
Table 1 Minimum squared distance for different modulation types with average power normalized to one

\begin{tabular}{ll}
\hline Modulation & $\boldsymbol{d}_{\boldsymbol{c}}^{\mathbf{2}}$ \\
\hline BPSK & 4 \\
QPSK & 2 \\
16-PSK & 0.1522 \\
$64-P S K$ & 0.0096 \\
16-QAM & 0.4 \\
64-QAM & 0.0952 \\
\hline
\end{tabular}

$$
\begin{aligned}
& p_{e-1{ }^{s t} \text { Stage }} \leq \int_{0}^{\infty} \exp \left(-\frac{M d_{c}^{2} \sigma_{d}^{2}}{4 N \sigma_{n}^{2}} q\right) p(q) \mathrm{d} q \\
& =\left[\frac{1}{2}\left(1-\sqrt{\frac{M d_{c}^{2} S N R}{4 N L+M d_{c}^{2} S N R}}\right)\right]^{L} \\
& \times \sum_{k=0}^{L-1}\left(\begin{array}{c}
L-1+k \\
k
\end{array}\right)\left[\frac{1}{2}\left(1+\sqrt{\frac{M d_{c}^{2} S N R}{4 N L+M d_{c}^{2} S N R}}\right)\right]^{k},
\end{aligned}
$$

where $\left(\begin{array}{l}a \\ b\end{array}\right)$ is the binomial coefficient, and $S N R=\frac{\sigma_{d}^{2}}{\sigma_{n}^{2}}$.

Assuming that no error is propagated from the first stage to the second stage, the second stage involves maximum likelihood estimation of the originally transmitted symbols from the $L$ branch outputs $\tilde{\mathbf{r}}_{l}^{\prime}$ in (14), each of which comes with diversity order 1 . The upper bound for this error probability is thus given by

$$
\begin{aligned}
& \left(p_{e-2^{\text {nd }} \text { Stage }}\left(1-p_{e-1^{s t} \text { Stage }}\right)\right) \\
& \leq\left[\frac{1}{2}\left(1-\sqrt{\frac{d_{c}^{2} S N R}{4 L+d_{c}^{2} S N R}}\right)\right]^{L} \\
& \times \sum_{k=0}^{L-1}\left(\begin{array}{c}
L-1+k \\
k
\end{array}\right)\left[\frac{1}{2}\left(1+\sqrt{\frac{d_{c}^{2} S N R}{4 L+d_{c}^{2} S N R}}\right)\right]^{k},
\end{aligned}
$$

Assuming that there is error propagated from the first stage to the second stage, we can rewrite (14) as following

$$
\begin{aligned}
& \tilde{\mathbf{r}}_{l}^{\prime}=\tilde{\mathbf{r}}_{z p}^{j}-\boldsymbol{\Pi}_{l} \mathbf{Q}_{z p} \hat{\mathbf{x}}_{M}^{j} \\
& =\mathbf{H}^{\prime} \mathbf{Q}_{z p} \mathbf{x}_{M}^{j}+\tilde{\mathbf{n}}_{N}^{j}-\boldsymbol{\Pi}_{l} \mathbf{Q}_{z p}\left(\mathbf{x}_{M}^{j}+\mathbf{n}_{1}\right) \\
& =\mathbf{H}_{l} \mathbf{Q}_{z p} \mathbf{x}_{M}^{j}+(\underbrace{\tilde{\mathbf{n}}_{N}^{j}-\Pi_{l} \mathbf{Q}_{z p} \mathbf{n}_{1}}_{\mathbf{n}_{2, l}}),
\end{aligned}
$$

where $\mathbf{n}_{1}$ has variance $\varepsilon_{\text {MMSE }}$ and $\mathbf{n}_{2, l}$ has variance $\sigma_{n}^{2}=\sigma_{n}^{2}+\sum_{t=1, t \neq l}^{L}|\mathbf{h}(l)|^{2} \varepsilon_{\text {MMSE }} \approx\left(1+\frac{N}{M}\right) \sigma_{n}^{2}$. Thus, the error probability for this scenario can be written as 


$$
d_{c}^{2}
$$

where $S N R^{\prime}=\frac{M}{M+N} S N R$. Therefore, (22) can be reconstructed following $(25,26)$, and $(28)$.

\section{Computational complexity analysis}

In this section, we focus on the computational complexity of the proposed receiver compared to the conventional MMSE-ZP-OFDM receiver. The computational complexity is measured based on the number of the complex multiplications and complex divisions. Since complex divisions can be implemented in various different ways, we did not decompose them into complex multiplication. Note that it is not trivial to elaborate explicit complexity expressions for different OFDM systems in terms of the system's parameters. Specifically, system's complexity depends on the parameters such as $N, M, D$, and $L$, each of which are decisive in finding the number of the complex multiplications involved. Finding a closed-form expression in terms of the aforementioned system parameters is rather difficult; therefore, in this work, we have derived expressions for the approximate multiplicative complexity counts and have used Matlab simulations to find the exact number of complex multiplications implemented. To do so, we have used the minimal multiplicative bounds [26] to find the arithmetic complexity of FFT of different block lengths. Besides, to implement the FFT of length $W$ decomposable to the product of two prime numbers $a$ and $b$, we have implemented $a$ FFTs of size $b$ or $b$ FFTs of size $a$ instead, without inquiring any additional operations such as multiplications by twiddle factors [27].

Following (5), we derive the approximate computational complexity count for the conventional MMSEZP-OFDM as below

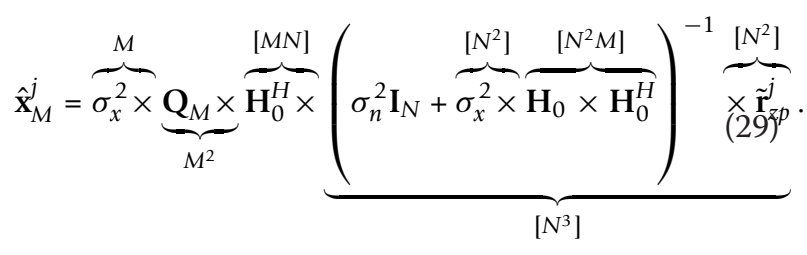

where [.] stands for the approximate operation count involved in the operation implementation. (28) requires total of $N^{3}+N^{2}(N+M+2)+M^{2}+M(N+1)$ operations.

Similarly, we derive the approximate computational complexity count for the low-complexity receiver using (11) and (16) as following

$$
\hat{\mathbf{x}}_{M}^{j}=\overbrace{\sigma_{x}^{2} \frac{\mathbf{N}}{M} \times}^{[M]} \underbrace{\mathbf{V}^{H} \times \overbrace{\boldsymbol{\Lambda}^{H} \times}^{[N]}}_{[N M]} \underbrace{(\frac{N}{M} \sigma_{n}^{2} \mathbf{I}+\overbrace{\sigma_{x}^{2} \times \overbrace{\boldsymbol{\Lambda}}^{[N]} \boldsymbol{\Lambda}^{H}}^{[N]})^{-1} \underbrace{\left.\times r_{[3}^{j} b^{\prime}\right)}_{[N]}}_{[N]}
$$

$$
\tilde{\mathbf{z}}^{j}=\frac{\overbrace{\mathbf{Q}_{z p}^{H} \times}^{[M N]}(\sum_{l=0}^{L} \overbrace{\mathbf{H}_{l}^{H} \times}^{[N]} \overbrace{\mathbf{z}_{l}^{j}}^{[N(M+5)+M]})}{\underbrace{\left.\sum_{l=0}^{L} \| N(M+6)+L M\right]}_{[M]}} .
$$

Therefore, the total number of operations required to implement the low-complexity receiver is $N((M+6)$ $L+M)+M(L+1)$.

We also derive the approximate computational complexity count for the M-ZP-OFDM receiver that includes the additional complexity brought to the transmitter side by multiplying the zero-padded information symbols by the set of matrices $\mathbf{C}_{2, l}$, as well as the computational complexity at the receiver side. Thus, using [19] we have

$$
\mathbf{r}_{c, l}=\mathbf{H}^{\prime} \overbrace{\Psi_{2, l} \mathbf{Q}_{z p}}^{\left[N^{2}\right]} \mathbf{x}_{M}^{j}+\mathbf{n}_{N^{\prime}}^{j}
$$

which shows the additional complexity cost at the transmitter side per diversity branch and

$$
\mathbf{r}_{c, l}^{\prime}=\underbrace{\Psi_{1, l} \mathbf{r}_{c, l},}_{\left[N^{2}\right]}
$$

$$
=\frac{\overbrace{\mathbf{Q}_{z p}^{H} \times}^{[M N]} \underbrace{(\sum_{l=0}^{L} \overbrace{\mathbf{H}_{l}^{H} \times}^{[N]} \overbrace{\left.\mathbf{r}_{c, l}^{\prime}\right]}^{\left[N^{2}\right]})}_{\left[L\left(N^{2}+N\right)\right]}}{\underbrace{\sum_{l=0}^{L}\left\|\mathbf{H}_{l}\right\|^{2}}_{[M]}}
$$

which shows the additional complexity cost at the receiver side. Therefore, the total number of operations required to implement the low-complexity receiver is $N$ $(N L+L+M)+M$.

The overall computational complexity for the LC-ZPOFDM receiver, $\mathrm{M}-\mathrm{ZP}$-OFDM receiver, and the conventional MMSE-ZP-OFDM receiver is summarized in Table 2 for the case of $M=64$ and $N=80$ and 
Table 2 Comparison of overall computational complexity

\begin{tabular}{lllll}
\hline Implementation & \multicolumn{2}{l}{ Number of complex multiplications } & \multicolumn{2}{l}{ Number of complex divisions } \\
& Scenario $\mathbf{1}$ & Scenario $\mathbf{2}$ & Scenario $\mathbf{1}$ & Scenario 2 \\
\hline ZP-OFDM-MMSE & 16,372 & 17,087 & NONE & NONE \\
LC-ZP-OFDM & 2,688 & 8,408 & 80 & 80 \\
M-ZP-OFDM & $R C=3,3408$ & $R C=66,648$ & NONE & NONE \\
& $\mathrm{TC}=25,600$ & $\mathrm{TC}=51,200$ & & \\
\hline
\end{tabular}

$T C$ transmitter complexity, $R C$ receiver complexity

scenarios with different combinations of channel memory lengths:

Scenario (1) $L+1=5$

Scenario (2) $L+1=10$

Note that the computational complexity of M-ZPOFDM receiver at the transmitter side does not include the CSI estimation. The computational complexity of the LC-ZP-OFDM receiver and the M-ZP-OFDM receiver versus the MMSE-ZP-OFDM receiver in terms of the number of the complex multiplications is illustrated in Figure 1, which is plotted against time, assuming that the channel estimate is updated at every $t$ seconds. We would like to emphasize that the MMSE equalizer in MMSE-ZP-OFDM requires the inversion of an $N \times N$ Toeplitz channel dependent matrix, which has to be computed at every $t$ seconds with a complexity of the order $O\left(N^{2}\right)$. Note that as the channel memory lengths of the underlying channel links increase, the complexity of MMSE-ZP-OFDM, M-ZP-OFDM, and the LC-ZPOFDM receiver increases.

It is observed from Figure 1 that for scenarios one and two with reasonable memory length for the underlying channels, the aggregate complexity of the LC-ZP-OFDM receiver is insignificant compared to the conventional

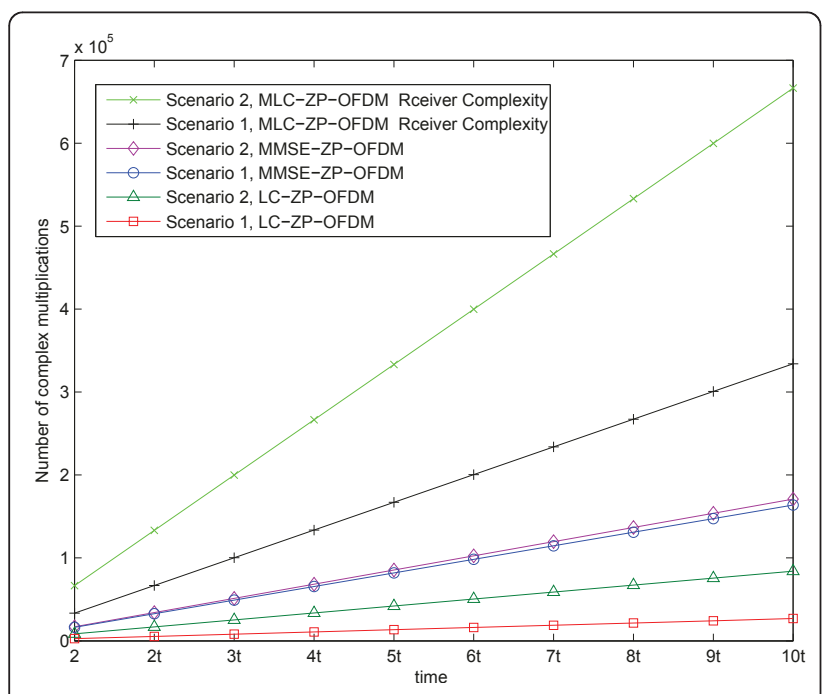

Figure 1 Computational complexity of the LC-ZP-OFDM receiver compared to MMSE-ZP-OFDM.
MMSE-ZP-OFDM as well as M-ZP-OFDM, as channel parameters change with time.

\section{Numerical results}

In this section, we present Monte-Carlo simulation results for the proposed receiver assuming a quasi-static Rayleigh fading channel.

Figure 2 illustrates the symbol error rate (SER) performance of the proposed receiver for $L+1=2$. We assume 64-QAM, $M=64$, and $N=80$ as is practiced in the context of HL2 systems. Our simulation results indicate that LC-ZP-OFDM receiver outperforms the conventional MMSE-ZP-OFDM equalizer by $\approx 3.3 \mathrm{~dB}$ without error propagation $(E P)$ from the first stage and by $\approx 0.5$ $\mathrm{dB}$ with EP from first stage at $\mathrm{SER}=10^{-3}$. We are also including the SER performance of the M-ZP-OFDM receiver with perfect CSI, as well as partial CSI (limited CSI is available, e.g., only one of the two channel taps are available at the transmitter side). As is illustrated in Figure 2, the M-ZP-OFDM receiver with partial CSI outperforms the conventional MMSE-ZP-OFDM by $\approx 4$ $\mathrm{dB}$ at SER $=10^{-3}$, when $l=1$. The system's SER performance for 64-QAM AWGN channel is provided as a reference curve.

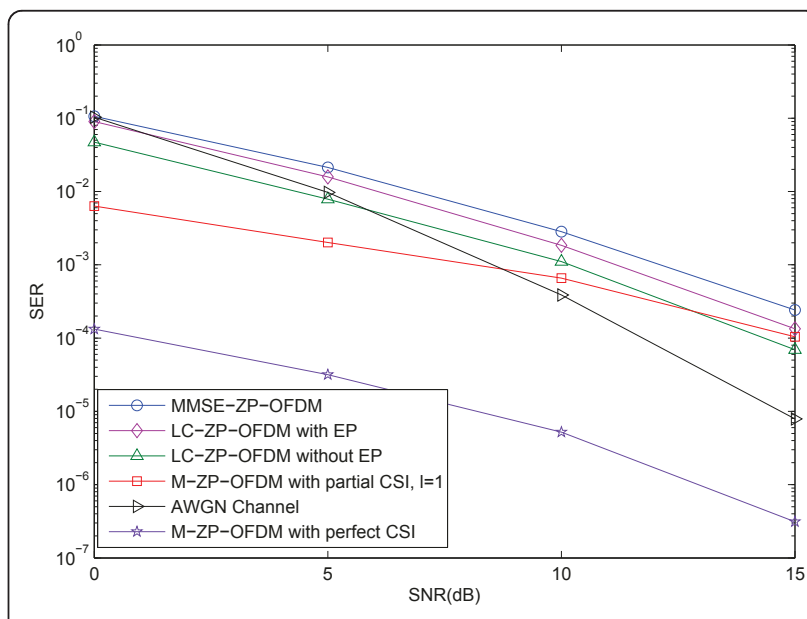

Figure 2 SER performance of MMSE-ZP-OFDM, LC-ZP-OFDM receiver with and without $E P$, and $M-Z P-O F D M$ in the context of HL2 $(L+1=2)$. 
In Figure 3, we provide further results on the performance of the LC-ZP-OFDM receiver for $L+1=6$. Our results indicate that the LC-ZP-OFDM receiver outperforms the MMSE-ZP-OFDM equalizer by $\approx 5 \mathrm{~dB}$ without $\mathrm{EP}$ and by $\approx 0.75 \mathrm{~dB}$ with $\mathrm{EP}$ at $\mathrm{SER}=10^{-7}$. Moreover, we are including the SER performance of the M-ZP-OFDM receiver with partial CSI (e.g., only two or three of the six channel taps are available at the transmitter side) when $l=2,3$. It can be seen that the proposed receiver outperforms the MMSE-ZP-OFDM receiver by $\approx 4 \mathrm{~dB}$ at $\mathrm{SER}=10^{-6}$, when $l=3$.

In Figure 4, the SER performance of the LC-ZP-OFDM receiver for $L+1=2,4,6$ is presented, assuming 64-QAM modulation in the context of HL2, and is compared with the theoretical values presented in Section V. As is illustrated in Figure 4, both the theoretical curves and their corresponding simulated versions come with similar slopes. As an example, when $L+1=4$, the theoretical curve differs from its simulated counterpart by $\approx 2 \mathrm{~dB}$.

\section{Conclusion}

We propose a novel reduced-complexity MMSE-based receiver for ZP-OFDM systems. We show that, by incorporating linear processing techniques, our MMSE-based receiver is able to collect full antenna and multipath diversity gains. Simulation results demonstrate that our LC-ZP-OFDM receiver outperforms the conventional MMSE-ZP-OFDM receiver, while maintaining much less complexity by avoiding channel dependent matrix inversion. By using linear processing techniques that require minimum computational complexity, the communication power is minimized at no additional hardware cost. We also propose a modified receiver that outperforms the conventional MMSE-ZP-OFDM.

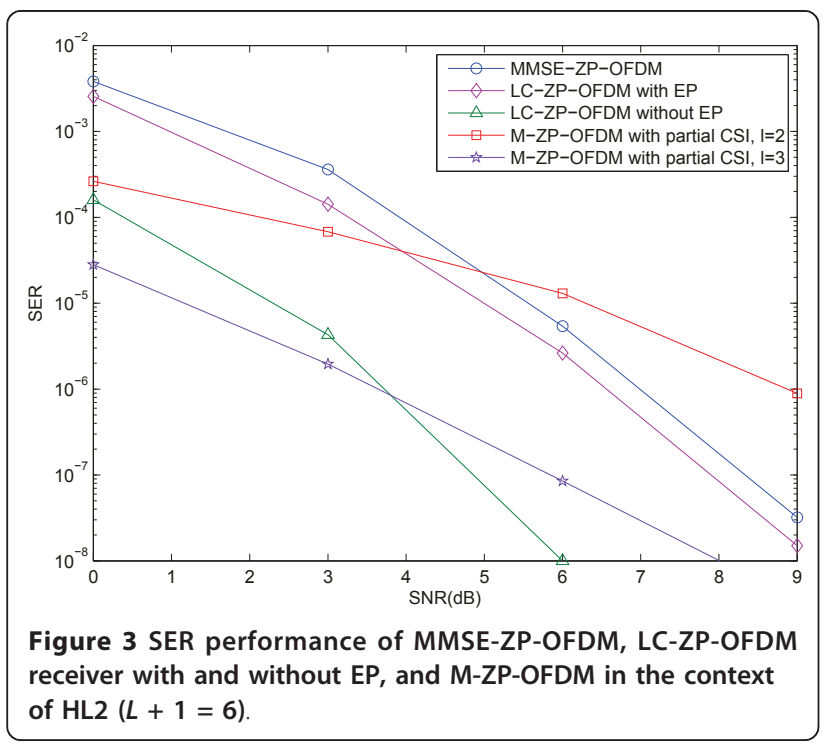

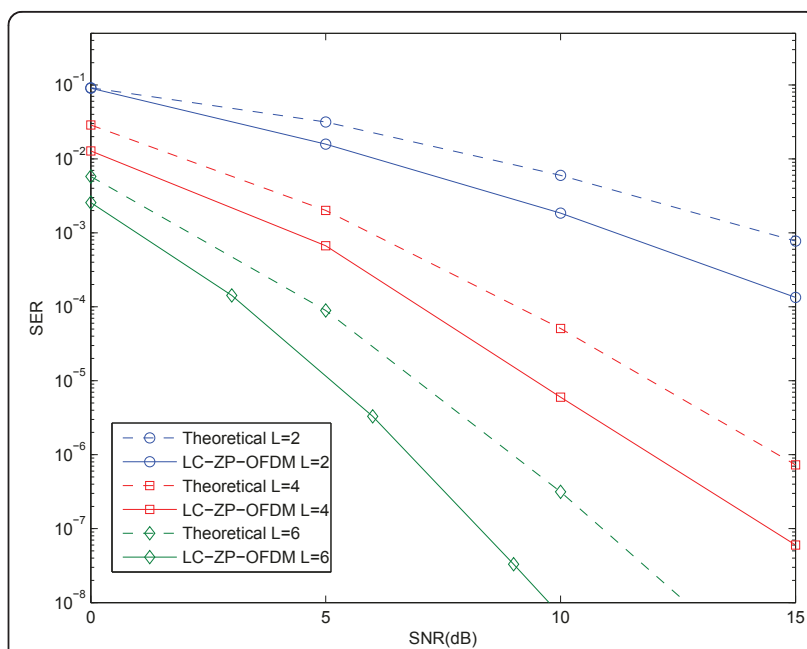

Figure 4 SER performance of LC-ZP-OFDM receiver compared to theoretical.

The authors declare that they have no competing interests.

\section{Endnotes}

Notation: $\overline{(.)},(.)^{\mathrm{T}},(.)^{\dagger}$ and $(.)^{\mathrm{H}}$ denote conjugate, transpose, pseudo inverse, and Hermitian transpose operations, respectively. $|$.$| denotes the absolute value, and$ |l. $\|$ denotes the Euclidean norm of a vector. [. $]_{k, l}$ denotes the $(\mathrm{k}, \mathrm{l})$ th entry of a matrix, $[.]_{k}$ denotes the kth entry of a vector, $\mathbf{I}_{M}$ denotes the identity matrix of size $M$, and $\mathbf{0}_{M \times M}$ denotes all-zero matrix of size $M \times$ $M$. Q represents the $M \times M$ FFT matrix whose $(1, \mathrm{k})$ element is given by $\mathbf{Q}(l, k)=1 / \sqrt{M} \exp (j 2 \pi l k / M)$ and $\mathbf{Q}_{M}^{-1}=\mathbf{Q}_{M}^{H}$ represents the $M \times M$ IFFT matrix where $0 \leq$ $l, k \leq M-1$. Bold upper-case letters denote matrices and bold lower-case letters denote vectors.

\section{Note}

${ }^{0}$ The work of S. Muhaidat was supported in part by the Natural Sciences and Engineering Research Council (NSERC) of Canada under grant RGPIN372049.

Received: 19 December 2010 Accepted: 25 September 2011

Published: 25 September 2011

References

1. Z Wang, GB Giannakis, Linearly precoded or coded OFDM against wireless channel fades?, in Proceedings of the IEEE Workshop on Signal Processing Advances for Wireless Communications, Taiwan, China (2001)

2. WW Lu, Compact multidimensional broadband wireless: the convergence of wireless mobile and access. IEEE Commun. Mag, 119-123 (2000)

3. ETSI Normalization Committee, in Digital Broadcasting Systems for Television, Sound and Data Services; Framing Structure, Channel Coding and Modulation for Digital Terrestrial Television. Norme ETSI, document pr ETS 300 744, (European Telecommunications Standards Institute, Sophia-Antipolis, Valbonne, 1996) 
4. ANSI T1E1.4 Committee Contribution, in The DWMT: A Multicarrier Transceiver for ADSL using M-band Wavelets, Technical Report, ANSI, (1993)

5. Broadband Radio Access Networks (BRAN), in HIPERLAN Type 2 Technical Specification Part 1-Physical Layer. Eur. Telecommun. Standards Inst. (ETSI), DTS/BRAN030 003-1 (1999)

6. DP Palomar, JM Cioffi, MA Lagunas, Joint Tx-Rx beamforming design for multicarrier MIMO channels: a unified framework for convex optimization. IEEE Trans. Signal Process. 51(9) (2003)

7. W Zou, Y Wu, COFDM: an overview. IEEE Trans. Broadcast. 41, 1-8 (1995). doi:10.1109/11.372015

8. D Divsalar, MK Simon, Multiple trellis coded modulation (MTCM). IEEE Trans. Commun. 36, 410-419 (1988). doi:10.1109/26.2765

9. AJ Viterbi, Error bounds for convolutional codes and an asymptotically optimum decoding algorithm. IEEE Trans. Inform. Theory IT-13, 260-269 (1967)

10. I Lee, Space-time bit-interleaved coded modulation for OFDM systems. IEEE Trans. Signal Process. 52, 820-825 (2004). doi:10.1109/TSP.2003.822350

11. Z Liu, Y Xin, GB Giannakis, Linear constellation precoding for OFDM with maximum multipath diversity and coding gains. IEEE Trans. Commun. 51, 416-427 (2003). doi:10.1109/TCOMM.2003.809791

12. J Boutros, E Viterbo, Signal space diversity: a power- and bandwidthefficient diversity technique for the Rayleigh fading channel. IEEE Trans. Inform. Theory. 44, 1453-1467 (1998). doi:10.1109/18.681321

13. AO Hero, TL Marzetta, Cutoff rate and signal design for the quasistatic Rayleigh fading space-time channel. IEEE Trans. Inform. Theory. 47, 2400-2416 (2001). doi:10.1109/18.945254

14. Y Rong, SA Vorobyov, AB Gershman, Linear block precoding for OFDMsystems based on maximization of mean cutoff rate. IEEE Trans. Signal Process. 53(12), 4691-4696 (2005)

15. V Le Nir, M Helard, Reduced-complexity space-time block coding and decoding schemes with block linear precoding. IEE Electron. Lett. 39(14), 1066-1068 (2003). doi:10.1049/el:20030671

16. V Le Nir, M Helard, R Le Gouable, Space-time block codes with maximal diversity in OFDM-based systems, in Proceedings of the 5th World Wireless Congress (WWC'2004) San Francisco, USA (2004)

17. B Muquet, Z Wang, GB Giannakis, M de Courville, P Duhamel, Cyclic prefixing or zero padding for wireless multicarrier transmissions?. IEEE Trans. Commun. 50, 2136-2148 (2002). doi:10.1109/TCOMM.2002.806518

18. MS Alouini, A Goldsmith, Adaptive modulation over Nakagami channels. J. Wirel. Commun. 13(1-2), 119-143 (2000)

19. A Goldsmith, S Chua, Variable rate variable power M-QAM for fading channels. IEEE Trans. Commun. 45, 1218-1230 (1997). doi:10.1109/26.634685

20. T Keller, L Hanzo, Adaptive multicarrier modulation: a convenient framework for time-frequency processing in wireless communications. Proc. IEEE. 88, 611-640 (2000). doi:10.1109/5.849157

21. GG Raleigh, JM Cioffi, Spatio-temporal coding for wireless communication. IEEE Trans. Commun. 46, 357-366 (1998). doi:10.1109/26.662641

22. T Keller, L Hanzo, Adaptive multicarrier modulation: a convenient framework for time-frequency processing in wireless communications. Proc. IEEE. 88, 611-640 (2000). doi:10.1109/5.849157

23. K Wong, R Cheng, K Letaief, R Murch, Adaptive antennas at the mobile and base stations in an OFDM/TDMA system. IEEE Trans. Commun. 49, 195-206 (2001). doi:10.1109/26.898262

24. O Edfors, M Sandell, J-J van de Beek, SK Wilson, PO Briesson, OFDM channe estimation by singular value decomposition. IEEE Comm. Lett. 46(7), 931-939 (1998)

25. A Gusmo, R Dinis, N Esteves, On frequency-domain equalization and diversity combining for broadband wireless communications. IEEE Commun. Lett. 51(7), 1029-1033 (2003)

26. MT Heideman, C Sidney Burrus, On the number of multiplications necessary to compute a length-2n DFT. IEEE Trans. Acoust. Speech. Sig. Proc. 34, 91-95 (1986). doi:10.1109/TASSP.1986.1164785

27. P Duhamel, M Vetterli, Fast Fourier transforms: a tutorial review and a state of the art. Signal Process, 19, 259-299 (1990)

doi:10.1186/1687-1499-2011-109

Cite this article as: Eghbali and Muhaidat: A new reduced-complexity detection scheme for zero-padded OFDM transmissions. EURASIP Journal on Wireless Communications and Networking 2011 2011:109.

\section{Submit your manuscript to a SpringerOpen ${ }^{\mathcal{O}}$ journal and benefit from:}

- Convenient online submission

- Rigorous peer review

- Immediate publication on acceptance

- Open access: articles freely available online

- High visibility within the field

- Retaining the copyright to your article

Submit your next manuscript at $\gg$ springeropen.com 